\title{
Exploration of the Drosophila buzzatii transposable element content suggests underestimation of repeats in Drosophila genomes
}

\author{
Nuria Rius ${ }^{1 *}$, Yolanda Guillén ${ }^{1}$, Alejandra Delprat ${ }^{1}$, Aurélie Kapusta ${ }^{2}$, Cédric Feschotte $^{2}$ and Alfredo Ruiz ${ }^{1}$
}

\begin{abstract}
Background: Many new Drosophila genomes have been sequenced in recent years using new-generation sequencing platforms and assembly methods. Transposable elements (TEs), being repetitive sequences, are often misassembled, especially in the genomes sequenced with short reads. Consequently, the mobile fraction of many of the new genomes has not been analyzed in detail or compared with that of other genomes sequenced with different methods, which could shed light into the understanding of genome and TE evolution. Here we compare the TE content of three genomes: D. buzzatii st-1, j-19, and D. mojavensis.

Results: We have sequenced a new D. buzzatii genome (j-19) that complements the $D$. buzzatii reference genome (st-1) already published, and compared their TE contents with that of D. mojavensis. We found an underestimation of TE sequences in Drosophila genus NGS-genomes when compared to Sanger-genomes. To be able to compare genomes sequenced with different technologies, we developed a coverage-based method and applied it to the $D$. buzzatii st-1 and j-19 genome. Between 10.85 and $11.16 \%$ of the D. buzzatii st- 1 genome is made up of TEs, between 7 and $7,5 \%$ of D. buzzatii j-19 genome, while TEs represent $15.35 \%$ of the D. mojavensis genome. Helitrons are the most abundant order in the three genomes.

Conclusions: TEs in D. buzzatii are less abundant than in D. mojavensis, as expected according to the genome size and TE content positive correlation. However, TEs alone do not explain the genome size difference. TEs accumulate in the dot chromosomes and proximal regions of D. buzzatii and D. mojavensis chromosomes. We also report a significantly higher TE density in D. buzzatii and D. mojavensis X chromosomes, which is not expected under the current models. Our easy-to-use correction method allowed us to identify recently active families in D. buzzatii st-1 belonging to the LTR-retrotransposon superfamily Gypsy.
\end{abstract}

Keywords: Drosophila, Buzzatii, Transposable elements, Genome

\section{Background}

Transposable elements (TEs) are mobile DNA sequences present in virtually all the eukaryote genomes sequenced and account for variable fractions of the genomes they inhabit. TEs are important not only because of their abundance but also because they are active components of

*Correspondence: nuria.rius.camps@gmail.com

1 Department de Genética i Microbiologia, Universitat Autònoma de Barcelona, Bellaterra (Barcelona), Spain

Full list of author information is available at the end of the article the genomes, inducing structural rearrangements, inactivating or duplicating genes and adding or removing regulatory regions $[1]$.

There are two classes of TEs, those that mobilize via an RNA intermediate belong to class I and those which transpose directly, leaving the donor site, or via a DNA intermediate, to class II $[2,3]$. Further divisions in this classification comprise orders that distinguish TEs with different insertion mechanisms, and superfamilies that are composed of TEs with similar domain structures and protein sequences. 
Progress in all aspects of genome sequencing and assembly has driven a revolution in the field. After D. melanogaster [4] and D. pseudoobscura [5] were sequenced, joint efforts provided the research community with the genomes of ten new Drosophila species which allowed multiple species comparisons [6]. These 12 genomes were sequenced with Sanger technology. After those, six de novo genomes were published individually [7-12], and eight more together [13]; these 14 genomes were sequenced mainly with Next-Generation Sequencing (NGS) technology.

The production of new genomes seems unstoppable and the comparisons and the knowledge drawn from them limitless. However, the information contained in some de novo draft genomes sequenced with NGS is not fully accurate $[14,15]$. TEs, because of their repetitive nature, are at the root of most of the problems that cause misassemblies [16, 17]. Hence, contextualization and comparison of the TE fraction of genomes sequenced and annotated separately is difficult and scarce. The latest advances in sequencing technology $[18,19]$ and standardization in annotation methods [20] may contribute to solve this issue, but meanwhile, sequenced genomes keep piling up.

In this article, we analyze in detail the TE content of the D. buzzatii reference (st-1) genome [12], and compare it to that of a second D. buzzatii strain ( $\mathrm{j}-19)$, described here, and that of $D$. mojavensis, another member of the repleta group [6]. We also compare the TE fraction in all available Drosophila genus genomes to test whether there are differences between NGS and Sanger-sequenced genomes, propose a method to correct such differences, and apply it to the genomes of two strains of $D$. buzzatii.

\section{Methods}

\section{Genomes}

The genomes used in this work were all freely available online except the genome of $D$. buzzatii strain $\mathrm{j}-19$, which is described here and available through http://dbuz.uab. cat.

Strain j-19 was isolated from flies collected in Ticucho (Argentina) using the balanced-lethal stock Antp/ $\Delta^{5}[21]$. Individuals of the $\mathrm{j}-19$ strain are homozygous for the chromosome arrangement $2 j$ [22]. DNA was extracted from male and female adults using the sodium dodecyl sulfate (SDS) method [23] or the method described by Piñol et al. [24] for isolating high molecular weight DNA. Three Illumina HiSeq Paired End (PE) libraries were prepared and sequenced at CNAG (Centro Nacional de Análisis Genómico) with an insert size of $500 \mathrm{bp}$ and a mean read length of $102 \mathrm{bp}$. SOAPdenovo [25] version 1.05 was used to assemble the genome of the j-19 strain. We fed the assembler with $251,719,776$ filtered reads setting the assembler with kmer size $k=31$. The final assembly contains 10529 scaffolds over $3 \mathrm{~kb}$ (total size $=153,440,896 \mathrm{bp}$ ).
The N50 index is 1666, and the N50 length $24268 \mathrm{bp}$, the N90 index is 6825, and the N90 length $5747 \mathrm{bp}$.

Publicly available genomes from the Drosophila genus were downloaded from FlyBase $(D$. ananassae r1.3, $D$. erecta $\mathrm{r} 1.3, D$. grimshawi $\mathrm{r} 1.3, D$. melanogaster $\mathrm{r} 6.05, D$. mojavensis $\mathrm{r} 1.3$, D. persimilis $\mathrm{r} 1.3$, D. pseudoobscura $\mathrm{r} 3.2$, D. sechellia $\mathrm{r} 1.3$, D. simulans $\mathrm{r} 1.3$ and $\mathrm{r} 2.01$ [26], D. virilis $\mathrm{r} 1.2$, D. willistoni $\mathrm{r} 1.3$, and D. yakuba r1.3 [6]), NCBI (D. albomicans [7], D. biarmipes, D. bipectinata, D. elegans, D. eugracilis, D. ficusphila, D. kikkawai, D. miranda [8], D. rhopaloa, D. suzukii [10], and D. takahashii [13]) or project web sites $(D$. americana $\mathrm{H} 5$ (http://cracs.fc.up. pt/ nf/dame/index.html) [11] and D. buzzatii st-1 (http:// dbuz.uab.cat) [12]).

\section{Transposable element library}

We built a custom library to annotate and classify the mobile elements in the D. buzzatii and D. mojavensis genomes. The library comprised already known repeats (FlyBase and Repbase) and de novo elements found in the D. buzzatii st-1 genome (RepeatModeler and Repclass). FlyBase's canonical set of TEs (http://flybase.org/) were blasted [27] against an early assembly of the D. buzzatii st-1 genome. For each query, significant hits were manually inspected in order to recover the most complete copy. Repbase [28] repeats from Insecta species were added to the library. RepeatModeler (version 1.0.4) [29] was used with RepeatScout [30] and Recon [31] to identify repeats, and the RMBlast engine and Repbase database to classify them. Repclass [32] was used to classify repeats identified by RepeatScout. Elements classified by Repclass as being distinct from previously identified repeats, or as being more complete, were added to the library. Sequences classified as simple, satellite or low complexity repeats, were removed from the library. Additionally, a blast analysis was performed to filter non-TE related sequences. Sequences with significant hits (e-value blast $<1 \mathrm{e}-25$ ) with D. mojavensis coding sequences (cds) and at the same time with no significant similarity to repeats deposited in Repbase were removed.

\section{Repeat annotation}

To compare the three genomes of the two Drosophila repleta group species (D. buzzatii st-1, D. buzzatii j-19 and D. mojavensis), we masked them with RepeatMasker [33] (version 4.0.5) and RMBlast (version 2.2.27+) and the $D$. buzzatii custom library using the default options except for cut off (score value 250), nolow and norna. We used the RepeatMasker output files ".out to estimate the amount of nucleotides of each order and superfamily. We also used RepeatMasker, with cut off 250, nolow, and norna, to assess the TE content of the 27 available Drosophila genomes, from 25 species. To reduce library bias factor we used the RepBase Insecta library. The assembly size 
was used, in each case, to compute the percentage of transposable elements.

\section{Chromosomal analysis}

We analyzed the TE distribution along the chromosomes of $D$. buzzatii st-1 and D. mojavensis. We used the previously mapped and oriented scaffolds, the 158 N90 scaffolds (145 Mb) of D. buzzatii [12], and the 11 N80 scaffolds $(156 \mathrm{Mb})$ of $D$. mojavensis [34]. These scaffolds are the longest scaffolds that cover the 90 and $80 \%$ of the entire assemblies of $D$. buzzatii st-1 and D. mojavensis respectively. Consequently, the shortest scaffolds which had not been mapped and are presumably the TE-richest could not be included in this analysis. The mapped scaffolds were broken down into $50 \mathrm{~kb}$ non-overlapping windows using bedtools (makewindows) and the TE nucleotides in each window were calculated using also bedtools (intersect). We plotted the TE density (TE bp/window length) for all windows, including those smaller than $50 \mathrm{~kb}$ from the tip of each scaffold, in the reported order.

To assess the TE-density in every chromosome, in the proximal regions and in the rest of the chromosome independently, another set of windows was made with the $D$. buzzatii and D. mojavensis mapped scaffolds previously mentioned. The most proximal $3 \mathrm{Mb}$ of chromosomes $\mathrm{X}$, $2,3,4$ and 5 ( $\sim 10 \%$ of the chromosome) were divided in $50 \mathrm{~kb}$ windows as well as the remaining $\sim 90 \%$ of the chromosomes, and the entire chromosome 6 . Only whole windows $(50 \mathrm{~kb})$ were taken into account. For each chromosome and region, we computed the mean TE-density and standard deviation and plotted the TE-density window distribution. Additionally, differences among these distributions (whole chromosome, proximal and central+distal regions) were tested with the two-sample KolmogorovSmirnov test.

\section{Correction}

We mapped the reads used in the genome pre-assembly of D. buzzatii st-1 (21924977 reads from 454, Illumina, and Sanger) [12] with GS Reference Mapper (v2.9) (http://454. com/products/analysis-software) to the final D. buzzatii assembly using the default options. GS Reference Mapper aligned $95.3 \%$ of the reads (20422434 reads), 20270 reads less than those used by gs-Assembler to build the pre-assembly. We also mapped the D. buzzatii j-19 Illumina reads to the D. buzzatii $\mathrm{j}-19$ with Bowtie2. Every read base pair that mapped to a TE-annotated position was added up to calculate the coverage of the position. The corrected value for each TE order and superfamily is the sum of read base pairs annotated as part of that order or superfamily, divided by the average coverage. D. buzzatii st- 1 average coverage is the genes average coverage, $22.37 x$, calculated with the same procedure used for the TEs, but with 13657 genes identified in D. buzzatii st-1 genome [12]. The average coverage for D. buzzatii $\mathrm{j}-19$ is 160x, SOAPdenovo estimation.

\section{Results}

TE content in $D$. buzzatii and $D$. mojavensis assemblies

In D. buzzatii st- 1 , TEs account for $8.43 \%$ of the assembly, about twice the value of TEs in D. buzzatii j-19 (4.15\%), but almost half of the value of D. mojavensis (15.35\%). In order to make a fair comparison, we also considered only 3-kb or longer scaffolds for D. mojavensis, 2419 (187.4 Mb) out of 6841 scaffolds (193.8 Mb). However, the TE fraction in D. mojavensis genome is still higher $(14.35 \%)$ than the fraction in both $D$. buzzatii strains. Henceforth, the complete $D$. mojavensis genome assembly was used for the subsequent analyses.

The contribution of the different orders, defined by Wicker et al. [2], to the total amount of TEs (Fig. 1 and Table 1), is similar between the two D. buzzatii genomes (Helitrons, LINEs, LTR-retrotransposons, TIRtransposons, and Mavericks/Polintons), and differs from the $D$. mojavensis one. Despite the similarities, there are some differences. Although Helitrons are the most abundant order in the three genomes, they are more abundant in the D. buzzatii st- 1 genome ( $40.61 \%$ of the TEs content) than in the other two genomes (30.65\% in D. buzzatii j-19 and $33.90 \%$ in D. mojavensis). LTR-retrotransposons are the second most abundant order in $D$. mojavensis (33.46\%), but not in D. buzzatii (17.38 \% in st-1 and $19.54 \%$ in $j$-19) where in both strains LINEs are the second most abundant order in genome contribution. TIRtransposons are more frequent in $D$. buzzatii genomes (14.81 \% in st- 1 and $14.46 \%$ in $\mathrm{j}-19)$ than in D. mojavensis (9.24\%), like the unclassified repeats that are more abundant in D. buzzatii (7.15\% in st-1 and $9.11 \%$ in $\mathrm{j}-19$ ) than in D. mojavensis (2.42\%).

\section{Chromosomal distribution}

The TE distribution along D. buzzatii N90 mapped scaffolds and D. mojavensis N80 mapped scaffolds (Fig. 2) shows a similar pattern in both species: increased TE density in (i) chromosome 6 (the "dot" chromosome), (ii) the pericentromeric regions of all chromosomes, and (iii) chromosome X compared with the autosomes (Fig. 2). The density of the main orders plotted individually (Additional file 1: Figure S1a-h) reveals the prevalence of Helitrons in D. buzzatii proximal regions, specially the 3 $\mathrm{Mb}$ closest to the centromere.

We compared the abundance of TEs annotated in $D$. buzzatii and D. mojavensis, specifically the distribution of TE density in $50 \mathrm{~kb}$ windows, for whole chromosomes (the N90 mapped scaffolds of D. buzzatii and the N80 mapped scaffolds of $D$. mojavensis), for proximal regions ( $3 \mathrm{Mb}$ ), and for central and distal regions (Table 2). It is important to note that only the largest scaffolds are being considered, 


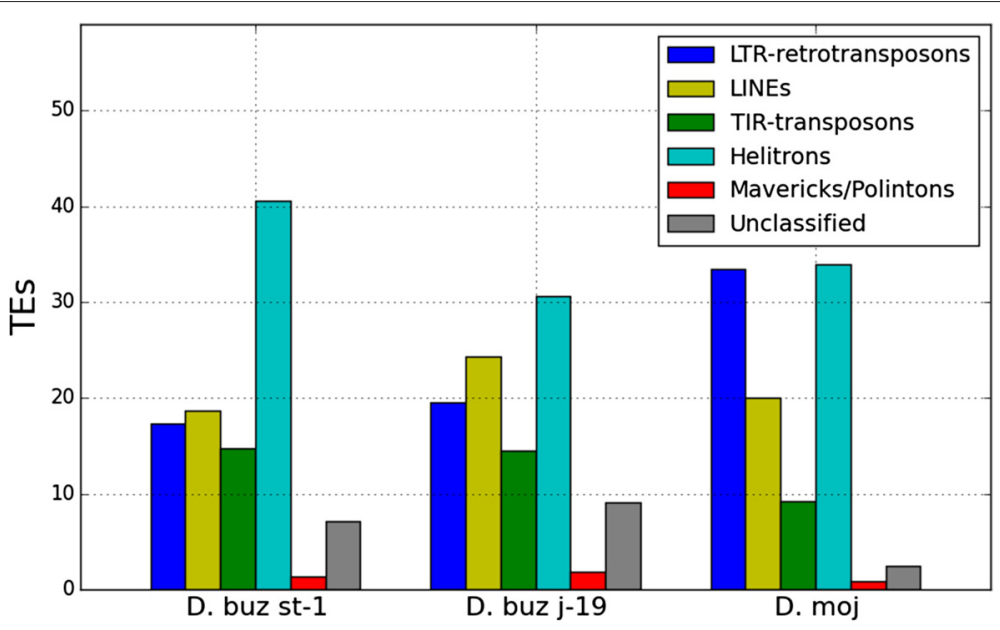

Fig. 1 TE Order abundance. Percentage of transposable element orders relative to the mobile fraction of the genomes of $D$. buzzatii st-1, j-19, and $D$. mojavensis

and that 10 and $20 \%$ of $D$. buzzatii and D. mojavensis assemblies respectively, contained in the smallest and typically TE-enriched scaffolds, were discarded from this analysis. This explains the differences between the annotation of the whole assembly and the mean values of the mapped scaffolds. The smaller and TE-richer scaffolds are likely located in proximal regions, as the centromeric regions have the higher TE-density and more nested TEs. However, all recent TE insertions are susceptible to misassemblies and small scaffolds could be located between mapped scaffolds.

D. mojavensis chromosomes, as a whole, or any of their parts, have a higher TE fraction than D. buzzatii chromosomes. The biggest differences are in the proximal regions, diminishing in the central and distal regions. Chromosome 6 (Muller element F) is the TE-richest chromosome in both species, $41.22 \%$ in D. buzzatii and $46.30 \%$ in D. mojavensis. In D. buzzatii, $8.32 \%$ of chromosome X (Muller element A) is made up by TEs, followed by the other chromosomes with values between 4.80 and $5.86 \%$. In D. mojavensis, the $\mathrm{X}$ chromosome has $11.81 \%$ of TEs, chromosome $310.70 \%$ and the rest of the chromosomes have values between 8.14 and $6.06 \%$. D. buzzatii chromosomes 6 and $\mathrm{X}$, when analyzed as a whole, are the only ones with TE density distributions significantly different (two-sample Kolmogorov-Smirnov test $p<0.001$ ) from all other chromosomes, whereas in D. mojavensis it is chromosomes 6, X, and 3 (Additional file 2: Tables S1, S2, S3 and S4) that show significant differences. If we discard the 3 most proximal $\mathrm{Mb}$ and chromosome 6 , chromosome $\mathrm{X}$ of both species is the only one with significantly different TE density distribution from all the other chromosomes (Additional file 2: Tables S5, S6, S7 and S8). When the pericentromeric regions are compared, in D. buzzatii there are not significant differences among chromosomes, while among $D$. mojavensis proximal regions, chromosome $3 \mathrm{TE}$ density is significantly different from the rest of the chromosomes (Additional file 2: Tables S9, S10, S11 and S12). Consequently, in both species, chromosomes 6 and X display a significantly different TE distribution pattern from the rest of the chromosomes.

\section{Impact of the sequencing method in Drosophila genus}

Because the genomes of $D$. mojavensis, $D$. buzzatii st-1 and j-19 strains were sequenced with different platforms and assembly strategies (see Methods), the differences in TE content between these genomes could be related to the methodologies used. More specifically, the Sanger sequenced $D$. mojavensis genome [6] shows a higher TE content than the D. buzzatii reference (st-1) genome sequenced with 454, Illumina and Sanger [12], which itself has a higher TE content than the D. buzzatii $\mathrm{j}-19$ genome sequenced only with Illumina. Therefore it seems that NGS yields a smaller repeat content than Sanger sequencing [35].

In order to test this hypothesis, we widened our scope to include all the available genomes of Drosophila genus (Table 3). As in the cases of D. mojavensis and D. buzzatii there is a difference in the mobile fraction depending on the sequencing method. The mean TE percentage in the 12 genomes sequenced with Sanger technology is $19.31 \%$, whereas that in the 15 newly sequenced genomes (chiefly produced using NGS) is $10.98 \%$. The differences are significant (Mann-Whitney U-test $p$-value $=0.001421)$ and clear when the values are plotted (Fig. 3).

It is possible that the species sequenced with Sanger technology have per se more TEs than those sequenced with NGS, and sequencing or assembly methods do not influence the assembly TE fraction. However, when species belonging to the same subgroup are compared, the 
Table 1 TE contribution of every order and superfamily (kb) to the D. buzzatii (st-1 and j-19 before and after the correction) and D. mojavensis genomes ${ }^{\mathrm{a}}$

\begin{tabular}{|c|c|c|c|c|c|}
\hline \multirow[t]{2}{*}{ Superfamily } & \multicolumn{4}{|c|}{ D. buz } & \multirow[t]{2}{*}{ D. moj } \\
\hline & st-1 & st-1 corr. & $j-19$ & j-19 corr. & \\
\hline \multirow{2}{*}{ LTR Total } & 2366.44 & 4693.31 & 1243.43 & 2050.57 & 9953.02 \\
\hline & (17.38\%) & (26.03 \%) & (19.54 \%) & (17.62 \%) & (33.46 \%) \\
\hline BelPao & 435.35 & 1025.76 & 198.65 & 432.82 & 2255.95 \\
\hline Copia & 309.80 & 522.62 & 162.75 & 275.82 & 718.71 \\
\hline ERVK & 10.92 & 9.97 & 8.09 & 7.52 & 18.06 \\
\hline Gypsy & 1610.37 & 3134.95 & 873.94 & 1334.42 & 6960.30 \\
\hline \multirow{2}{*}{ LINE Total } & 2541.65 & 3401.72 & 1551.05 & 2221.12 & 5977.29 \\
\hline & (18.66 \%) & (18.87 \%) & (24.37 \%) & (19.08 \%) & $(20.09 \%)$ \\
\hline CR1 & 396.35 & 761.48 & 117.39 & 546.88 & 947.96 \\
\hline I & 74.63 & 136.15 & 20.19 & 38.59 & 110.53 \\
\hline Jockey & 478.24 & 600.72 & 246.54 & 345.78 & 765.64 \\
\hline L1 & 6.71 & 6.01 & 6.70 & 5.63 & 8.08 \\
\hline L2 & 191.37 & 213.18 & 145.73 & 148.74 & 395.99 \\
\hline LOA & 1.18 & 1.31 & 0.82 & 0.65 & 1.95 \\
\hline R1 & 1383.35 & 1663.22 & 1011.77 & 1133.23 & 3721.30 \\
\hline R2 & 1.49 & 9.30 & 0.51 & 0.38 & 23.03 \\
\hline R4 & 1.57 & 0.80 & 0.70 & 0.57 & 1.37 \\
\hline RTE & 6.76 & 9.55 & 0.69 & 0.68 & 1.43 \\
\hline \multirow{2}{*}{ TIR Total } & 2016.98 & 2476.88 & 919.50 & 1820.64 & 2747.83 \\
\hline & (14.81\%) & (13.74 \%) & (14.46 \%) & (15.64 \%) & (9.24\%) \\
\hline hAT & 563.03 & 661.13 & 239.06 & 414.90 & 654.13 \\
\hline Mutator & 21.00 & 16.32 & 16.14 & 14.05 & 22.73 \\
\hline Novosib & 17.35 & 16.43 & 11.89 & 10.77 & 16.15 \\
\hline$P$ & 590.70 & 830.17 & 216.28 & 713.43 & 752.39 \\
\hline PIF/Harbinger & 3.81 & 9.71 & 2.21 & 2.45 & 7.82 \\
\hline piggyBack & 18.67 & 9.46 & 5.38 & 5.79 & 77.21 \\
\hline Tc1/mariner & 407.93 & 507.35 & 186.38 & 363.43 & 534.42 \\
\hline Transib & 281.27 & 115.97 & 172.40 & 211.64 & 627.54 \\
\hline TIR other & 113.23 & 310.35 & 69.75 & 84.18 & 55.43 \\
\hline \multirow{2}{*}{ Helitron } & 5531.01 & 6331.89 & 1950.81 & 4689.50 & 10083.94 \\
\hline & (40.61 \%) & (35.12 \%) & (30.65 \%) & (40.29 \%) & $(33.90 \%)$ \\
\hline \multirow{2}{*}{ Maverick } & 189.27 & 129.44 & 118.57 & 100.34 & 263.81 \\
\hline & (1.39\%) & (0.72\%) & (1.86\%) & $(0.86 \%)$ & (0.89\%) \\
\hline \multirow{2}{*}{ Others } & 0.24 & 0.11 & 0.67 & 0.40 & 0.19 \\
\hline & (0\%) & (0\%) & (0\%) & (0\%) & (0\%) \\
\hline \multirow{2}{*}{ Unknown } & 973.76 & 994.61 & 580.02 & $756.66 c$ & 721.26 \\
\hline & (7.15\%) & (5.52\%) & (9.11\%) & (6.50\%) & (2.42\%) \\
\hline Total & 13619.34 & 18027.96 & 6364.04 & 11639.23 & 29747.33 \\
\hline
\end{tabular}

arder contributions, relative to the total TE fraction, are given in percentages

${ }^{b}$ Order total values are shown in boldface

Sanger-sequenced genomes show a consistently higher percentage of TEs. The mulleri subgroup species, $D$. buzzatii and $D$. mojavensis, have different values than those yielded by our custom library but the pattern is the same.
More examples (Table 3) are in the virilis, the ananassae or the obscura subgroups, where the species sequenced with shorter reads have a lower percentages of mobile elements. Two genomes from the virilis subgroup have 

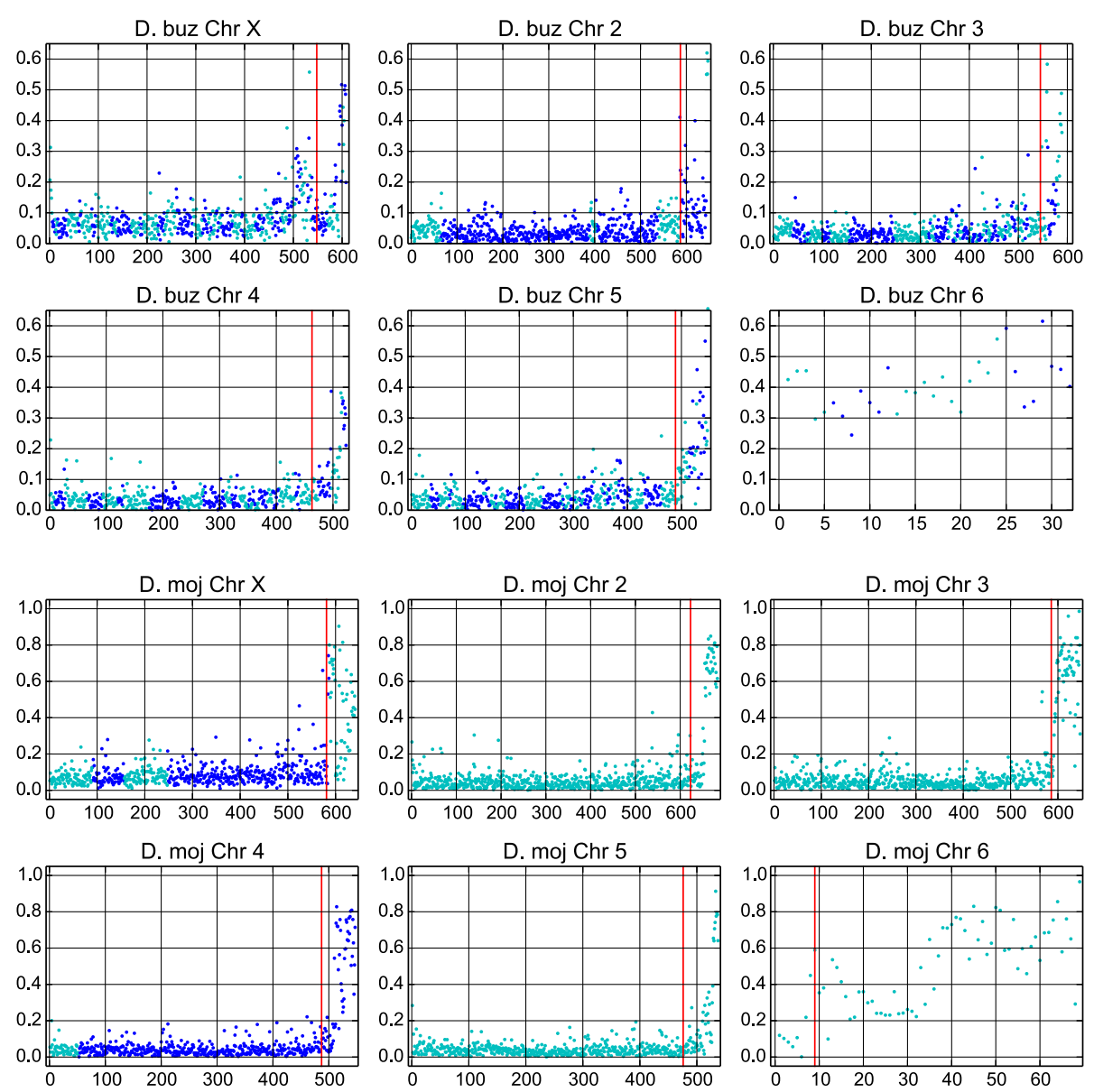

Fig. 2 Chromosomal TE density. Density of transposable elements in 50 kb non-overlapping windows, starting (left) from the telomere. Only mapped and oriented scaffolds are included, N90 for D. buzzatii st-1, and N80 for D. mojavensis. Changes in dot colors denote scaffold changes and the red lines mark the most proximal $3 \mathrm{Mb}$ of each chromosome

been sequenced, $D$. virilis with Sanger and $D$. americana with NGS, and have 17.51 and $9.11 \%$ of TEs respectively. D. ananassae sequenced with Sanger has $30.33 \%$ of TEs, D. bipectinata sequenced with NGS has $16.94 \%$. Similarly, D. persimilis and D. pseudoobscura, sequenced with Sanger technology, have 23.91 and $12.68 \%$ respectively, whereas D. miranda, sequenced with NGS, has $5.47 \%$ of TEs in its genome. Moreover, the case of the same species sequenced by both technologies further supports the trend. $D$. simulans has been recently resequenced with NGS and old Sanger sequences to amend significant problems with the previous Sanger project. Our results show that the newly sequenced genome has $8.44 \%$ of TEs (6.85\% according to $\mathrm{Hu}$ et al. [26], the authors of the latter assembly) while the old assembly has $11.85 \%$. Although various methodologies of repeat detection render various results, the use of the same procedure on Sanger and primarily NGS genomes gives consistently higher values of repeats in Sanger genomes. Hence, to accurately compare the results of $D$. buzzatii genome to other Sanger genomes like $D$. mojavensis, we thought it was necessary to correct our previous estimates of the D. buzzatii TE fraction.

\section{Correction of TE estimation by coverage}

We found $403.3 \mathrm{Mb}$ of reads, out of $3609 \mathrm{Mb}$, mapping to regions annotated as TEs in $D$. buzzatii st- 1 assembly, corresponding to $11.16 \%$ of all reads mapped. After dividing this $403.3 \mathrm{Mb}$ by the average gene coverage (22.37x) we got the corrected value of TEs of D. buzzatii, $18 \mathrm{Mb}$. Therefore there is a 1.32 fold underestimation $(4.4 \mathrm{Mb})$ with respect to the $13.6 \mathrm{Mb}$ initially annotated with RepeatMasker. If we keep considering the assembly size as the genome size, and assume the extra $4.4 \mathrm{Mb}$ belong to the gaps within scaffolds $(15 \mathrm{Mb})$ the initial estimate of TEs in the genome of $8.43 \%$ increases to $11.16 \%$. On the other hand, if we add the 4.4 new $\mathrm{Mb}$ to the assembly size, we get a genome size of $165.9 \mathrm{Mb}$ and the TE fraction is $10.85 \%$. The correction, also applyed to D. buzzatii j-19 genome, revealed that TEs correspond to $11.64 \mathrm{Mb}$ instead of the 6.4 $\mathrm{Mb}$ annotated, that means an increase from 4.15 to 
Table 2 TE fraction in D. buzzatii and D. mojavensis computed in 50 kb non-overlapping windows ${ }^{\mathrm{a}}$

\begin{tabular}{|c|c|c|c|c|c|c|c|}
\hline \multirow[t]{2}{*}{ Chr } & \multirow[t]{2}{*}{ Species } & \multicolumn{2}{|c|}{ Proximal } & \multicolumn{2}{|c|}{ Cent+Dist } & \multicolumn{2}{|c|}{ Total } \\
\hline & & TE (\%) & $\mathrm{N}$ & $\mathrm{TE}(\%)$ & $\mathrm{N}$ & TE (\%) & $\mathrm{N}$ \\
\hline \multirow[t]{2}{*}{$X$} & D. buzzatii & 16.13 & 57 & 7.44 & 505 & 8.32 & 562 \\
\hline & D. mojavensis & 42.24 & 59 & 8.71 & 579 & 11.81 & 638 \\
\hline \multirow[t]{2}{*}{2} & D. buzzatii & 13.91 & 59 & 4.77 & 638 & 5.54 & 697 \\
\hline & D. mojavensis & 38.68 & 60 & 5.11 & 622 & 8.06 & 682 \\
\hline \multirow[t]{2}{*}{3} & D. buzzatii & 12.96 & 58 & 4.12 & 522 & 5.01 & 580 \\
\hline & D. mojavensis & 60.52 & 60 & 5.60 & 586 & 10.70 & 646 \\
\hline \multirow[t]{2}{*}{4} & D. buzzatii & 12.50 & 58 & 3.77 & 434 & 4.80 & 492 \\
\hline & D. mojavensis & 39.24 & 60 & 4.31 & 486 & 8.14 & 546 \\
\hline \multirow[t]{2}{*}{5} & D. buzzatii & 14.98 & 58 & 4.06 & 462 & 5.87 & 520 \\
\hline & D. mojavensis & 21.47 & 60 & 4.11 & 476 & 6.06 & 536 \\
\hline \multirow[t]{2}{*}{6} & D. buzzatii & 41.22 & 28 & - & - & 41.22 & 28 \\
\hline & D. mojavensis & 50.65 & 60 & 14.22 & 8 & 46.30 & 68 \\
\hline \multirow[t]{2}{*}{ Total } & D. buzzatii & 16.51 & 318 & 4.87 & 2561 & 5.86 & 2879 \\
\hline & D. mojavensis & 42.13 & 359 & 5.68 & 2757 & 8.87 & 3116 \\
\hline
\end{tabular}

a Proximal regions corresponds to the 3 most proximal Mb; Central+ Distal to the rest of the chromosome and Total to both parts. $\mathrm{N}$ stands for number of windows. Only mapped and oriented scaffolds are present, N90 for D. buzzatii, and N80 for D. mojavensis

$7.59 \%(7.05 \%$ if we add the new $6.4 \mathrm{Mb}$ to the genome size). We conclude that the TE fraction in D. buzzatii st-1 is between 10.85 and $11.16 \%$ and between 7.59 and $7.05 \%$ in D. buzzatii $\mathrm{j}-19$.

Consequently, the orders and superfamilies with a higher correction factor are the ones with copies missing in the assembly. The results (Fig. 4 and Table 1) show that LTR-retrotransposons are the most underestimated order in D. buzzatii st-1 annotation by a factor of 1.98 . At the superfamily level (Fig. 5), Gypsy and BelPao are the most underestimated in D. buzzatii st-1 annotation, increasing after the correction by more than two fold.

$D$. buzzatii st- 1 and $D$. mojavensis TE profiles are more similar to each other after the correction as D. buzzatii LTR-retrotransposons have now overtaken LINEs as the second most frequent order. LINEs are underrepresented in the genome annotation by a factor of 1.34 . The superfamilies CR1 and R1 increase by 365 and $280 \mathrm{~kb}$ respectively after the correction. The $\mathrm{R} 2$ superfamily represents a singular case, since it is not relevant in absolute value (1.5 kb annotated), but the correction factor is the highest of all superfamilies (6.24 fold) and, after the correction, $9.3 \mathrm{~kb}$ are found to belong to the $\mathrm{R} 2$ superfamily. TIR-transposons are underestimated in the annotation by a 1.23 factor, with most superfamilies having a fair representation (correction factor close to one), but due to its large size, this small factor correction represent a substantial change in the base count. After the correction, the $\mathrm{P}$ superfamily sequence increased by $239 \mathrm{~kb}$ (1.41 fold), Tc1/mariner cover 99 new kb (1.24 fold) and hAT $98 \mathrm{~kb}$
(1.17 fold). Helitrons are underestimated by a 1.15 factor, but like TIR-transposons, their abundance in the genome prior to the correction ( 5.5 annotated $\mathrm{Mb}$ ) translates into a remarkable increase, $800 \mathrm{~kb}$ absent from the annotation. The correction, applyied to $D$. buzzatii j-19 reveals that Helitrons are heavily underrepresented in the annotation, while the LTR-retrotransposons are not as underestimated as in D. buzzatii st-1 (Table 1 and Additional file 1: Figures S2 and S3). Among superfamilies P, Helitron, and BelPao are the more underestimated in D. buzzatii j-19 assembly, by 3.3, 2.4 and 2.18 factors respectively. Gypsy superfamily is also remarkable if we look at the amount of new sequences with 460 new $\mathrm{Kb}$. These superfamilies are likely to include highly similar insertions probably recently transposed.

\section{Discussion and conclusions}

We have shown that $D$. buzzatii st-1 and j-19 genomes have a lower TE percentage than $D$. mojavensis. We have also reported that there is an underestimation of the mobile fraction of genomes sequenced with Next Generation Sequencing, possibly due to sequencing and assembly methods, which affect $D$. buzzatii st-1 genome, and j-19.

We have proposed a method based on read coverage to assess the magnitude of the bias, and used it to correct the D. buzzatii st- 1 and j-19 TE estimates. In D. buzzatii st- 1 the correction revealed another $4.4 \mathrm{Mb}$ of TEs and increased the TE percentage to $11 \%$, while for $D$. buzzatii $\mathrm{j}-19$ five new $\mathrm{Mb}$ of TEs were found, meaning TEs are $7 \%$ of the genome. Thus, although the TE content in 
Table 3 Percentage of TEs annotated with repeat masker and RepBase Insecta library on every available genomes of Drosophila genus

\begin{tabular}{|c|c|c|c|c|c|}
\hline Species & Subgenus & Group & Subgroup & Seq method & TEs \\
\hline D. albomicans & Drosophila & immigrans & nasuta & NGS & 2.73 \\
\hline D. buzzatii st-1 & Drosophila & repleta & mulleri & NGS & 5.99 \\
\hline D. buzzatii j-19 & Drosophila & repleta & mulleri & NGS & 2.40 \\
\hline D. mojavensis & Drosophila & repleta & mulleri & Sanger & 16.14 \\
\hline D. americana & Drosophila & virilis & virilis & NGS & 9.11 \\
\hline D. virilis & Drosophila & virilis & virilis & Sanger & 17.51 \\
\hline D. grimshawi & Hawaian & grimshawi & grimshawi & Sanger & 15.86 \\
\hline D. ananassae & Sophophora & melanogaster & ananassae & Sanger & 30.33 \\
\hline D. bipectinata & Sophophora & melanogaster & ananassae & NGS & 16.94 \\
\hline D. elegans & Sophophora & melanogaster & elegans & NGS & 12.05 \\
\hline D. eugracilis & Sophophora & melanogaster & eugracilis & NGS & 13.67 \\
\hline D. ficusphila & Sophophora & melanogaster & ficusphila & NGS & 9.45 \\
\hline D. erecta & Sophophora & melanogaster & melanogaster & Sanger & 14.41 \\
\hline D. melanogaster & Sophophora & melanogaster & melanogaster & Sanger & 21.67 \\
\hline D. sechellia & Sophophora & melanogaster & melanogaster & Sanger & 20.90 \\
\hline D. simulans & Sophophora & melanogaster & melanogaster & Sanger & 11.85 \\
\hline D. simulans & Sophophora & melanogaster & melanogaster & NGS & 8.44 \\
\hline D. yakuba & Sophophora & melanogaster & melanogaster & Sanger & 21.98 \\
\hline D. kikkawai & Sophophora & melanogaster & montium & NGS & 11.95 \\
\hline D. rhopaloa & Sophophora & melanogaster & rhopaloa & NGS & 18.62 \\
\hline D. biarmipes & Sophophora & melanogaster & suzukii & NGS & 14.48 \\
\hline D. suzukii & Sophophora & melanogaster & suzukii & NGS & 18.70 \\
\hline D. takahashii & Sophophora & melanogaster & takahashii & NGS & 14.68 \\
\hline D. miranda & Sophophora & obscura & obscura & NGS & 5.47 \\
\hline D. persimilis & Sophophora & obscura & obscura & Sanger & 23.97 \\
\hline D. pseudoobscura & Sophophora & obscura & obscura & Sanger & 12.68 \\
\hline D. willistoni & Sophophora & willistoni & willistoni & Sanger & 24.35 \\
\hline
\end{tabular}

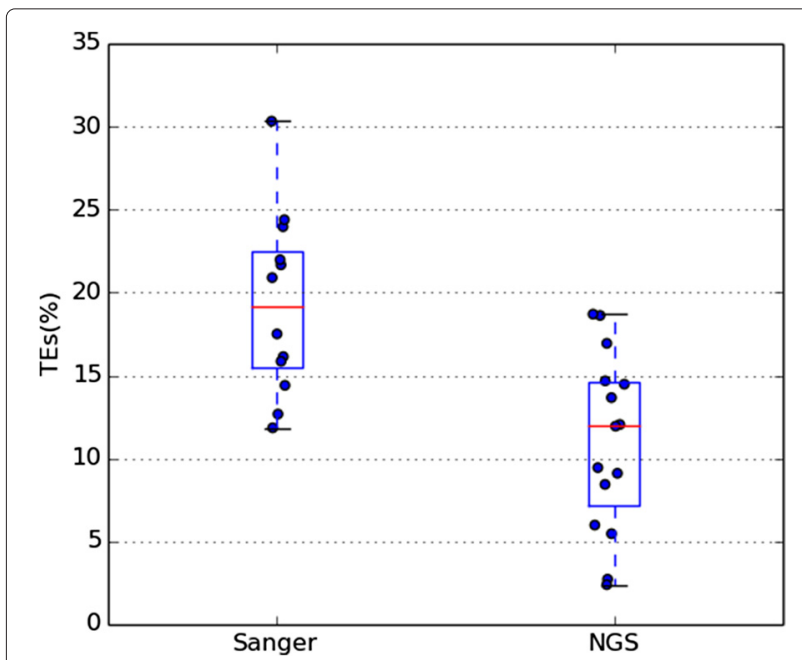

Fig. 3 TEs in Sanger and NGS genomes. Boxplot representing the TE \% in Drosophila genomes
D. buzzatii genome increased with the correction, it is still lower than that of $D$. mojavensis genome. Our methodology does not allow us to locate the TEs absent from the assembly. However, we consider it is important to describe the TEs present in the published assembly for several reasons. The differences while affecting particularly some orders and superfamilies have a small effect in others. Moreover, D. buzzatii uncorrected TE chromosomal distribution shows the same trends than those we observed in D. mojavensis. Finally, the published assembly should be analyzed and its limitations assessed in order to become a useful resource.

\section{$D$. buzzatii and $D$. mojavensis assembly TE content}

Our results show that TEs in D. buzzatii genome are less abundant than in D. mojavensis genome, even after taking into account the bias correction. The size of the two genomes have been estimated by Feulgen Image Analysis Densitometry and the $D$. buzzatii genome estimates are 


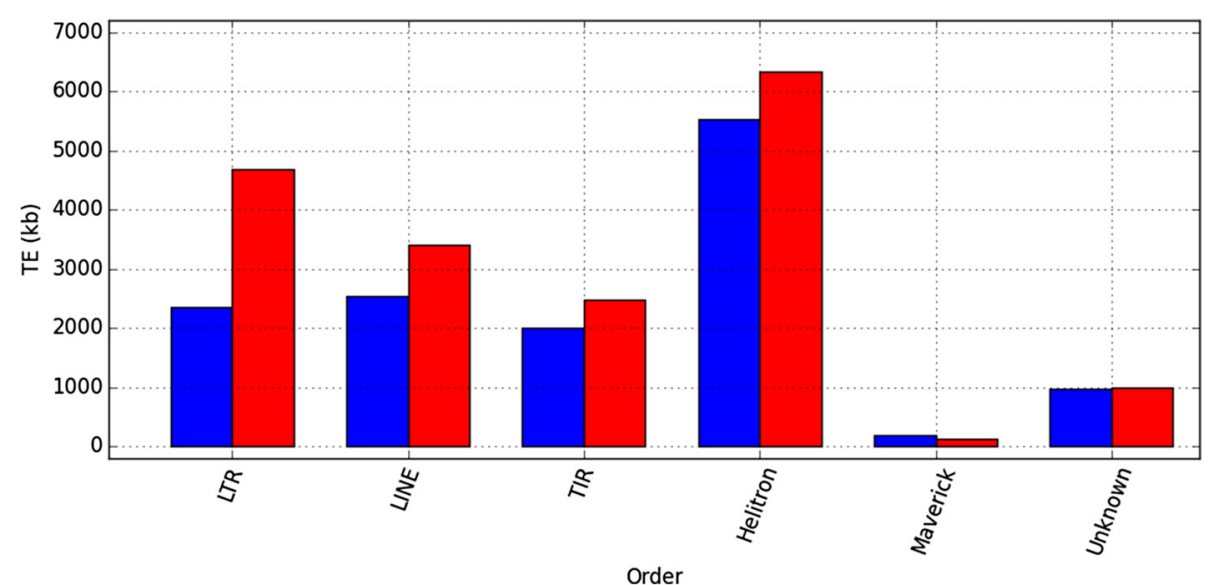

Fig. 4 Order correction. Main order contribution (kb) to D. buzzatii st-1 genome, before (blue) and after (red) the coverage-based correction

between $21 \%$ (st-1) and $25 \%$ ( $\mathrm{j}-19)$ smaller than those for D. mojavensis. Thus, our results agree with the well known positive correlation between genome size and transposable element fraction [36-38]. However, the difference in $T E$ content does not explain the difference in size between the two genomes. Interestingly, after the coverage-based correction applied to D. buzzatii st-1, the contribution of each order to the total TE content is more similar to that of $D$. mojavensis, suggesting that the changes that lead to the differences affected every order in a uniform manner.

There are several non-mutually excluding explanations for the wide diversity in genome sizes and the forces driving its variation. The mutational explanation, ascribe part of such diversity to differences in insertion and deletion rates among species $[39,40]$; other authors suggest that non-adaptative forces have diminished the efficiency of selection, explaining genome expansions [41]; positive natural selection proposes that genome size constraints may be different depending of the lineage history [42]. According to Charlesworth and Barton [42], having a larger genome size may be advantageous, or at least not as strongly selected against, in some scenarios. Genome size has been reported to be negatively correlated with developmental rate, which is also negatively correlated with body size [43, 44]. Hence, species without a constrain on developmental time and favored by a larger body size may have accumulated more repetitive sequences than closer species with developmental time constraints.

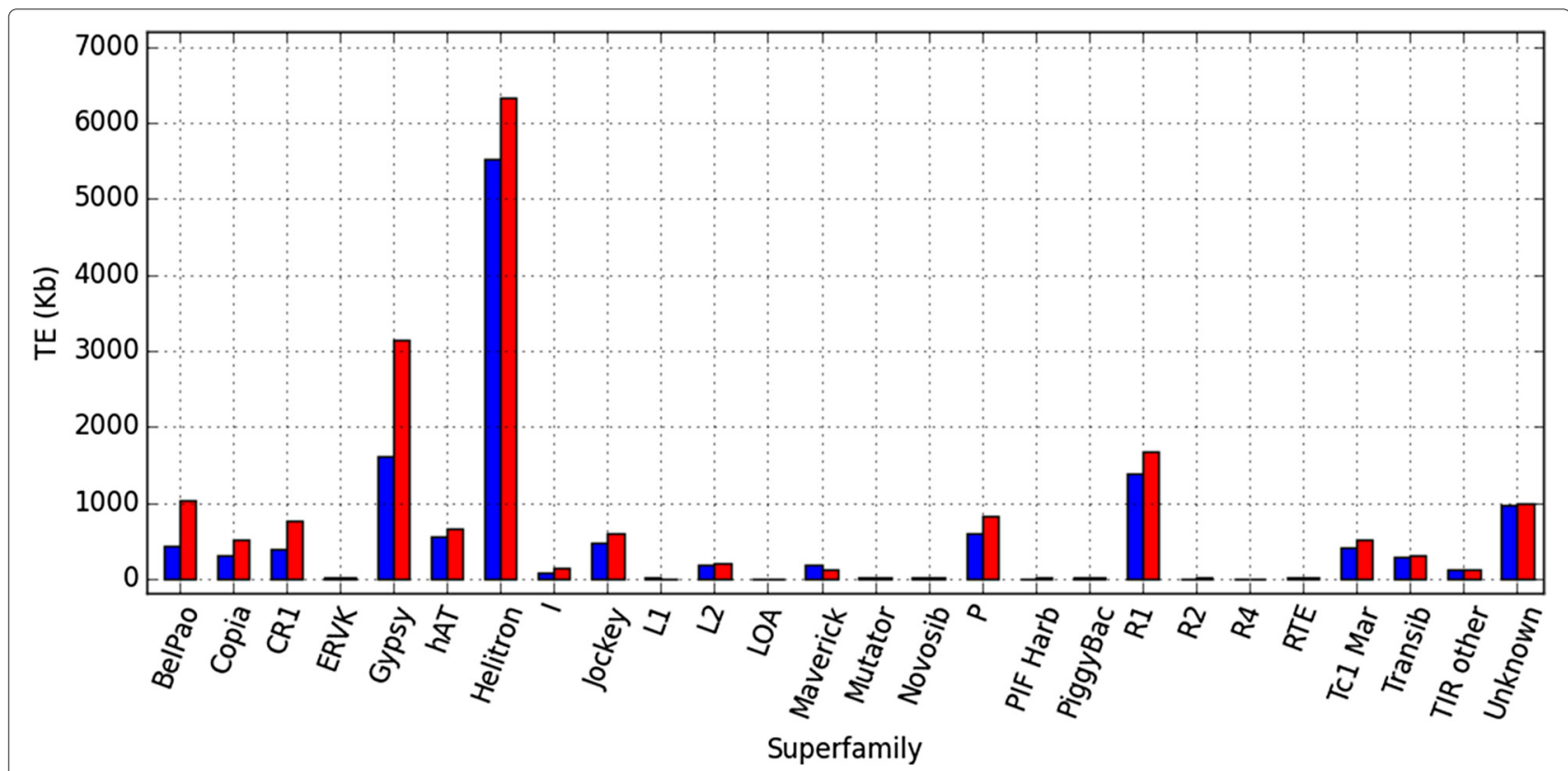

Fig. 5 Superfamily correction. Superfamily contribution (kb) to D. buzzatii st-1 genome before (blue) and after (red) the coverage-based correction 
This is possibly the case of D. buzzatii, which generally lay its eggs in rotting tissues of several Opuntia cacti, although it can occasionally use columnar cacti [45-47]; while $D$. mojavensis primarily uses larger rotting columnar or barrel cacti (Stenocereus gummosus and Stenocereus thurberi, and Ferocactus cylindraceous), except for the Santa Catalina Island population that uses Opuntia [48-51]. In other words, D. buzzatii individuals mainly live in smaller cacti which dry faster, consequently a more ephemeral resource than those used by $D$. mojavensis. The selective pressure to keep a faster development in $D$. buzzatii, or the relaxation of this pressure in D. mojavensis could be behind their different genome size and TE contribution.

\section{Chromosomal distribution of TEs}

TEs in D. melanogaster have been reported to accumulate in the proximal regions of the chromosomes, the transition between euchromatin and heterochromatin, where the recombination rate drops. The dot chromosome, which has a recombination rate considered null [52], has the highest TE density of all chromosomes [53, 54]. Moreover, recent analyses of several $D$. melanogaster populations have found a negative correlation between recombination rate and TE population frequency $[55,56]$.

TE dynamics has been extensively studied; however there is not a consensus about why some regions have a higher TE density. Ectopic recombination is so far the only explanation for the negative correlation between recombination rate and TE frequency. Recombination events involving non-homologous TE copies can lead to chromosomal rearrangements and inviable gametes [57]. According to the ectopic recombination hypothesis, the decrease in the recombination rates, seen in centromeric and telomeric regions, weakens the selection against TE insertions by reducing the crossing-over events between non-homologous TE copies [52, 58]. Accumulation of specific transposable elements in D. buzzatii centromeric regions was previously noticed using in situ hybridization $[59,60]$. Additionally $D$. mojavensis dot chromosome TE density has also been found to be higher than that of $D$. melanogaster, D. erecta and D. grimshawi [61]. We are now reporting $\mathrm{TE}$ accumulations in the dot chromosomes and in the proximal regions of the rest of the chromosomes of $D$. buzzatii st- 1 and $D$. mojavensis. The available linkage maps for $D$. buzzatii and D. mojavensis $[62,63]$ are not very detailed; even so, we can assume that like in $D$. melanogaster these regions have a reduced recombination rate.

The $\mathrm{X}$ chromosome poses a challenge when trying to explain its TE dynamics. Because the $\mathrm{X}$ has a higher recombination rate than the autosomes, and mutations are directly exposed to selection in hemizygous males, deleterious insertions should be removed more efficiently in the $\mathrm{X}$ chromosome than in the autosomes. An early analysis of the $D$. melanogaster reference genome showed a reduced accumulation of TEs in the D. melanogaster $\mathrm{X}$ chromosome [64]. However, recent analyses have surveyed several $D$. melanogaster populations and have not found evidence of a lower TE presence in the X chromosome, and some have even reported a higher abundance $[55,56,65]$. Our observations show that in $D$. buzzatii and $D$. mojavensis the $\mathrm{X}$ chromosome has a significantly higher TE density than the autosomes, except for the dot. And this difference remains even when the most proximal $3 \mathrm{Mb}$ are discarded. Interestingly, the increase is sustained throughout the whole length of chromosome $\mathrm{X}$ in both species (Fig. 2). The $\mathrm{X}$ higher TE density is observed not only in D. buzzatii but also in D. mojavensis. Consequently, the assembly problem, that could have more impact on chromosome $\mathrm{X}$ as using males and female flies implies a lower coverage, does not seem to explain our results. The argument that some families with an insertion preference for the $\mathrm{X}$ have recently suffered an expansion in D. melanogaster [65] is interesting and may suggest that $D$. buzzatii and D. mojavensis TEs are actively transposing. However, there are possibly other factors, besides recombination, needed to understand the unpredicted TE abundance in the $\mathrm{X}$ chromosome.

\section{TEs and NGS}

Issues with the NGS genomes repeats have been reported before [35] suggesting that stringent assembly strategies and shorter reads do not produce an accurate representation of the repeats in a specific locus but a consensus built with sequences from other loci [66]. Hence, the differences found in TE content between Sanger and NGS genomes are likely caused by an underestimation of NGS assembly methods rather than by an overestimation of TEs by Sanger technology. Although dealing with different technologies, it resembles the case of $D$. melanogaster Release 3 [67], where after extensive experimental efforts, most of the repetitive sequences of the previous release were found to be composite sequences of the newly sequenced TEs. It is also important to note that Sanger genomes, assembled with longer reads, may recover a longer fraction of the heterochromatin and go deeper in this region rich in repeated sequences than genomes sequenced with NGS. Consequently, comparing the mobile fraction of the two strains of D. buzzatii between them (st-1 sequenced with a mixture of Sanger, Illumina and 454 reads and j-19 sequenced solely with Illumina reads) and to D. mojavensis genome (sequenced with Sanger reads) raised questions about the reliability of such comparisons.

To find out if the sequencing technology, and potentially the assembly methods, implied major differences in TE annotation, we look at published genomes and their 
analyses of TE fractions. Two dozens of genomes of different Drosophila genus species have been released since $D$. melanogaster reference genome. Nevertheless, the mobile fraction of most of the recently published genomes has not been analyzed or has only been analyzed superficially $[7-9,11]$ yet there are some exceptions [10]. At least two analyses comparing some of these genomes in a uniform manner have been published $[6,9]$ but they yielded very different values. The main reasons seem to be the use of different annotation methods and updates in the TE libraries. The discrepancies between estimations compelled us to analyze all the Drosophila genus genomes available simultaneously, in the most homogeneous way possible and trying to reduce the unavoidable bias of library specificity. The values differ from previous studies but the comparisons should be more consistent. We found that genomes sequenced with Sanger technology have a higher TE percentage than those sequenced mainly with Illumina and 454 technologies. Because the data is not phylogenetically independent it is possible that species sequenced with one technology have actually a higher TE fraction than the ones sequenced with the other. However, from all the species from the same subgroup, sequenced with different technologies, the ones sequenced with Sanger show the highest TE percentage, suggesting that there is indeed an impact from the sequencing technology.

\section{Correction of $D$. buzzatii TE estimates}

We mapped the reads used in the D. buzzatii assembly back to the assembly, following the lead of several projects that used high quality reference genomes and re-sequenced data from different individuals to accurately identify TE insertions $[55,56,68,69]$. The mapping showed how some regions annotated as TE insertions had a TE coverage depth much higher than the surrounding regions. We also noticed that some gaps had TE annotations from the same family on each side, suggesting that the gap should be filled with TE sequence. In order to obtain a reliable estimate and account for the problems related to NGS (see above), we directly counted how many read nucleotides belonged to TEs. One could argue that some of those reads may belong to the heterochromatin, were casted aside during the assembly, and have been aligned now to euchromatin repeats. However, in D. buzzatii st-1 correction GS Reference Mapper aligned 20270 reads less in this process than those used by GS Reference Assembler. After mapping and dividing by the average coverage, we pulled the data for every order and superfamily together.

Sequence similarity among TE family copies is related to its transpositional activity. TE families which have recently transposed will contain highly similar copies and will be the most affected by the assembly problems mentioned before. Therefore, our correction method is expected to have a higher impact on these families. Our results show that LTR-retrotransposons were the most affected order by $D$. buzzatii st-1 correction. Their recent activity and their double repetitive nature, as not only LTR-retrotransposon copies will generate similar reads, but the LTRs from a single copy can produce reads susceptible to be assembled together are likely explanations. Additionally, LTR-retrotransposons are the longest TEs in Drosophila genomes, thus suffering more than other orders the artificial fragmentation by identification software [32] and assembly problems due to reads that do not span the lenght of the insertions. Osvaldo and Isis elements, from the Gypsy superfamily, were reported to be active in $D$. buzzatii [70,71], which agrees with our ours results as Gypsy is the LTR-retrotransposon superfamily with a higher correction rate for $D$. buzzatii st-1 and also a high rate for $D$. buzzatii $\mathrm{j}-19$. The LINEs superfamilies R1 and R2 are nested within ribosomal regions, typically poorly assembled, explaining their underestimation in $D$. buzzatii st-1 genome [72, 73].

D. melanogaster genome annotations and analyses of only euchromatic and both euchromatic and heterochromatic regions find the same order in the abundance of the major TE orders. According to [53, 74, 75] the contribution order is, from highest to lowest, LTR retrotransposons, LINE elements, TIR transposons, and Helitrons (when DINE-1 is annotated). This same order was found for most species in [6] work. However, it appears to be a difference in Drosophila subgenus order when Helitrons are taken into account. Yang and Barbash [76] carried out and extensive analysis of DINE-1 on the firsts 12 Drosophila genomes sequenced. Their analyses revealed that $D$. mojavensis is the second in number of DINE-1 copies, than those copies had probably undergone multiple rounds of transposition and silencing, and some had been recently transposed. Feschotte et al. [32] found that the $D$. melanogaster reported order was maintained in D. pseudoobscura and not in D. virilis, where Helitrons make up a higher fraction of the genome than TIR elements. This is in agreement with [9] observations for D. virilis and D. mojavensis, both from the Drosophila subgenus. Their analysis show how DNA elements, computing TIR elements and Helitrons together, are more abundant than LTR retrotransposons or LINE elements in these two species. Previous studies have already identified several families of Helitrons in D. buzzatii named $\mathrm{ISBu}$ (for Insertion Sequence of D. buzzatii) in chromosomal inversion breakpoints [77, 78]. We have now detected that over $800 \mathrm{~kb}$ of Helitrons were incorrectly assembled in D. buzzatii st-1, suggesting that $12.65 \%$ of the Helitrons have been recently transposed, while $5531 \mathrm{~kb}$ of Helitrons are either sequenced in reads with other regions, that allowed the assembler to map 
them, or are not as similar to confound the assembler. Helitrons are also the most abundant order in $D$. buzzatii $\mathrm{j}-19$ and is highly affected by the coveragebased correction. Hence, like in D. mojavensis, Helitrons seem to have undergone several rounds of activity and the TE content differences between Drosophila and Sophophora subgenera appear to be greater than initially thought.

Our methods has drawbacks; the correction does not inform of where the repeats are in the genome, or their specific sequence, an information that may not be precise in a NGS genome (see above). However, it is a method easy to apply that provides more acurate estimates of the abundance of each order and superfamily. Therefore, our strategy facilitates comparisons among the wealth of already sequenced genomes and deepens our understanding of genome evolution.

\section{Availability of data and material}

The datasets supporting the conclusions of this article are available in the Drosophila buzzatii genome project repository http://dbuz.uab.cat and within the article additional files.

\section{Ethics approval and consent to participate}

Not applicable.

\section{Consent for publication}

Not applicable.

\section{Additional files}

Additional file 1: Supplementary Figures. Supplementary Figure 1 (a to h). Chromosomal TE density. Main transposable element order density in $50 \mathrm{~kb}$ non-overlapping windows. Only mapped and oriented scaffolds are present, N90 scaffolds for D. buzzatii st-1 (a to d), and N80 scaffolds for D. mojavensis (e to h). Changes in dot colors denote scaffold changes. Supplementary Figure 2. D. buzzatii j-19 Order correction. Order contribution (kb) to D. buzzatii j-19 genome before (blue) and after (red) the coverage-based correction. Supplementary Figure 3. D. buzzatii j-19 Superfamily correction. Superfamily contribution (kb) to D. buzzatii j-19 genome before (blue) and after (red) the coverage-based correction. (ZIP $792 \mathrm{~kb}$ )

Additional file 2: Supplementary Tables. Supplementary Table 1. D statistics and $p$-values of $\mathrm{U}$ two-sample Kolmogorov-Smirnov tests comparing the distributions of TE densities in 50-Kb windows of each pair of chromosomes in three different sets: whole chromosome, central+distal and proximal regions. Only mapped and oriented scaffolds were considered. (PDF $389 \mathrm{~kb}$ )

\section{Abbreviations}

BSC: Barcelona supercomputing center; Cds: coding sequences; CNAG: Centro Nacional de Aná1 lisis Genómico; ISBu: insertion sequence of D. buzzatii; NGS: next-generation sequencing; PE: paired end; SDS: sodium dodecyl sulfate; TEs: transposable elements.

\section{Competing interests}

The authors declare that they have no competing interests.

\section{Authors' contributions}

NR designed, and carried out the transposable element analyses and drafted the manuscript. YG assembled D. buzzatii j-19 genome. AD extracted DNA for sequencing and contributed to the analyses design. AK helped with transposable element analyses. CF contributed to design the study. AR conceived of the study, participated in its design and coordination and helped to draft the final manuscript. All authors read and approved the final manuscript.

\section{Acknowledgments}

We want to thank Jordi Camps, Marta Gut and Ivo G Gut from the Spanish Centro Nacional de Análisis Genómico (CNAG) for their collaboration with sequencing of D. buzzatii j-19 and also to Valentí Moncunill and David Torrents from Barcelona Supercomputing Center (BSC) for their collaboration with the genome assembly.

\section{Funding}

This work was supported by grants BFU2008-04988 and BFU2011-30476 from the Spanish Ministerio de Ciencia e Innovación to A.R., grant R01GM077582 to C.F from the National Institutes of Health, and by PIF-UAB fellowship to N.R.

\section{Author details}

${ }^{1}$ Department de Genética i Microbiologia, Universitat Autònoma de Barcelona, Bellaterra (Barcelona), Spain. ${ }^{2}$ Department of Human Genetics, University of Utah School of Medicine, Salt Lake City, UT, USA.

Received: 22 October 2015 Accepted: 22 April 2016

Published online: 10 May 2016

\section{References}

1. Akagi K, Li J, Symer DE. How do mammalian transposons induce genetic variation? A conceptual framework: the age, structure, allele frequency, and genome context of transposable elements may define their wide-ranging biological impacts. BioEssays News Rev Mol Cellular Dev Biol. 2013;35(4):397-407. doi:10.1002/bies.201200133.

2. Wicker T, Sabot F, Hua-Van A, Bennetzen JL, Capy P, et al. A unified classification system for eukaryotic transposable elements. Nat Rev Genet. 2007;8(12):973-82. doi:10.1038/nrg2165. Accessed 30 Aug 2015.

3. Kapitonov W, Jurka J. A universal classification of eukaryotic transposable elements implemented in Repbase. Nat Rev Genet. 2008;9(5):411-2. doi:10.1038/nrg2165-c1. Accessed 30 Aug 2015.

4. Adams MD, Celniker SE, Holt RA, Evans CA, Gocayne JD, et al. The genome sequence of Drosophila melanogaster. Science (New York). 2000;287(5461):2185-95.

5. Richards S, Liu Y, Bettencourt BR, Hradecky P, Letovsky S, et al. Comparative genome sequencing of Drosophila pseudoobscura: chromosomal, gene, and cis-element evolution. Genome Res. 2005;15(1): 1-18. doi:10.1101/gr.3059305.

6. Drosophila 12 Genomes Consortium. Evolution of genes and genomes on the Drosophila phylogeny. Nature. 2007;450(7167):203-18. doi:10.1038/nature06341.

7. Zhou Q, Zhu H-M, Huang Q-F, Zhao L, Zhang G-J, et al. Deciphering neo-sex and $B$ chromosome evolution by the draft genome of Drosophila albomicans. BMC Genomics. 2012;13:109. doi:10.1186/1471-2164-13-109.

8. Zhou Q, Bachtrog D. Sex-specific adaptation drives early sex chromosome evolution in Drosophila. Science (New York). 2012;337(6092):341-5. doi:10.1126/science.1225385. Accessed 12 May 2015

9. Ometto L, Cestaro A, Ramasamy S, Grassi A, Revadi S, et al. Linking genomics and ecology to investigate the complex evolution of an invasive Drosophila pest. Genome Biol Evol. 2013;5(4):745-57. doi:10.1093/gbe/evt034

10. Chiu JC, Jiang X, Zhao L, Hamm CA, Cridland JM, et al. Genome of Drosophila suzukii, the spotted wing drosophila. G3 (Bethesda, Md.) 2013;3(12):2257-71. doi:10.1534/g3.113.008185.

11. Fonseca NA, Morales-Hojas R, Reis M, Rocha H, Vieira CP, et al. Drosophila americana as a model species for comparative studies on the molecular basis of phenotypic variation. Genome Biol Evol. 2013;5(4): 661-79. doi:10.1093/gbe/evt037.

12. Guillén Y, Rius N, Delprat A, Williford A, Muyas F, et al. Genomics of ecological adaptation in cactophilic Drosophila. Genome Biol Evol. 2015;7(1):349-66. doi:10.1093/gbe/evu291. 
13. Chen $Z X$, et al. Comparative validation of the $D$, melanogaster modENCODE transcriptome annotation. Genome Res. 2014;24(7): 1209-23. doi:10.1101/gr.159384.113.

14. Salzberg SL, Yorke JA. Beware of mis-assembled genomes. Bioinformatics (Oxford, England). 2005;21(24):4320-1. doi:10.1093/bioinformatics/bti769.

15. Narzisi G, Mishra B. Comparing De Novo genome assembly: the long and short of it. PLOS ONE. 2011;6(4):19175. doi:10.1371/journal.pone.0019175. Accessed 30 Aug 2015.

16. Ricker N, Qian H, Fulthorpe RR. The limitations of draft assemblies for understanding prokaryotic adaptation and evolution. Genomics. 2012;100(3):167-75. doi:10.1016/j.ygeno.2012.06.009.

17. Salzberg SL, Phillippy AM, Zimin A, Puiu D, Magoc T, et al. GAGE: a critical evaluation of genome assemblies and assembly algorithms. Genome Res. 2012;22(3):557-67. doi:10.1101/gr.131383.111.

18. English AC, Richards S, Han Y, Wang M, Vee V, et al. Mind the gap: upgrading genomes with pacific Biosciences RS long-read sequencing technology. PLoS ONE. 2012;7(11):47768.

doi:10.1371/journal.pone.0047768. Accessed 30 Aug 2015.

19. Huddleston J, Ranade S, Malig M, Antonacci F, Chaisson M, et al Reconstructing complex regions of genomes using long-read sequencing technology. Genome Res. 2014;168450-113. doi:10.1101/gr.168450.113. Accessed 30 Aug 2015.

20. McCoy RC, Taylor RW, Blauwkamp TA, Kelley JL, Kertesz M, et al. Illumina TruSeq synthetic long-reads empower de novo assembly and resolve complex, highly-repetitive transposable elements. PloS One. 2014;9(9): 106689. doi:10.1371/journal.pone.0106689.

21. Piccinali RV, Mascord LJ, Barker JSF, Oakeshott JG, Hasson E. Molecular population genetics of the alpha-esterase 5 gene locus in original and colonized populations of Drosophila buzzatii and its sibling Drosophila koepferae. J Mol Evol. 2007;64(2):158-70. doi:10.1007/s00239-005-0224-y.

22. Cáceres M, Puig M, Ruiz A. Molecular characterization of two natural hotspots in the Drosophila buzzatii genome induced by transposon insertions. Genome Res. 2001;11(8):1353-64. doi:10.1101/gr.174001.

23. Milligan BG. Total DNA Isolation In: Hoelzel AR, editor. Molecular Genetic Analysis of Population: A Practical Approach. 2nd Edition. New York, Tokyo: Oxford University Press; 1998.

24. Piñol J, Francino O, Fontdevila A, Cabré O. Rapid isolation of Drosophila high molecular weight DNA to obtain genomic libraries. Nucleic Acids Res. 1988;16(6):2736.

25. Li Y, Hu Y, Bolund L, Wang J. State of the art de novo assembly of human genomes from massively parallel sequencing data. Hum Genomics. 2010;4(4):271-7.

26. Hu TT, Eisen MB, Thornton KR, Andolfatto P. A second-generation assembly of the Drosophila simulans genome provides new insights into patterns of lineage-specific divergence. Genome Res. 2013;23(1):89-98. doi:10.1101/gr.141689.112. Accessed 04 June 2015.

27. Altschul SF, Madden TL, Schäffer AA, Zhang J, Zhang Z, et al. Gapped BLAST and PSI-BLAST: a new generation of protein database search programs. Nucleic Acids Res. 1997;25(17):3389-402.

28. Jurka J, Kapitonov W, Pavlicek A, Klonowski P, Kohany O, et al. Repbase Update, a database of eukaryotic repetitive elements. Cytogenet Genome Res. 2005;110(1-4):462-7. doi:10.1159/000084979.

29. Smit A, Hubley R. RepeatModeler Open-1.0. 2008. <http://www. repeatmasker.org $>$.

30. Price AL, Jones NC, Pevzner PA. De novo identification of repeat families in large genomes. Bioinformatics (Oxford, England). 2005;21 Suppl 1: 351-8. doi:10.1093/bioinformatics/bti1018.

31. Bao Z, Eddy SR. Automated de novo identification of repeat sequence families in sequenced genomes. Genome Res. 2002;12(8):1269-76. doi:10.1101/gr.88502.

32. Feschotte C, Keswani U, Ranganathan N, Guibotsy ML, Levine D. Exploring repetitive DNA landscapes using REPCLASS, a tool that automates the classification of transposable elements in eukaryotic genomes. Genome Biol Evol. 2009;1:205-20. doi:10.1093/gbe/evp023.

33. Smit A, Hubley R, Green P. RepeatMasker Open-3.0. 1996. <http://www. repeatmasker.org $>$.

34. Schaeffer SW, Bhutkar A, McAllister BF, Matsuda M, Matzkin LM, et al. Polytene chromosomal maps of 11 Drosophila species: the order of genomic scaffolds inferred from genetic and physical maps. Genetics. 2008;179(3):1601-55. doi:10.1534/genetics.107.086074.
35. Alkan C, Sajjadian S, Eichler EE. Limitations of next-generation genome sequence assembly. Nat Methods. 2011;8(1):61-5. doi:10.1038/nmeth.1527.

36. Kidwell MG. Transposable elements and the evolution of genome size in eukaryotes. Genetica. 2002;115(1):49-63.

37. Boulesteix $M$, Weiss $M$, Biémont C. Differences in genome size between closely related species: the Drosophila melanogaster species subgroup. Mol Biol Evol. 2006;23(1):162-7. doi:10.1093/molbev/msj012.

38. Feschotte $C$, Pritham EJ. DNA transposons and the evolution of eukaryotic genomes. Ann Rev Genet. 2007;41:331-68. doi:10.1146/annurev.genet.40.110405.090448.

39. Petrov DA, Sangster TA, Johnston JS, Hartl DL, Shaw KL. Evidence for DNA loss as a determinant of genome size. Science (New York). 2000;287(5455):1060-2.

40. Gregory TR. Insertion-deletion biases and the evolution of genome size. Gene. 2004;324:15-34.

41. Lynch M. The frailty of adaptive hypotheses for the origins of organismal complexity. Proc Nat Acad Sci. 2007;104(suppl 1):8597-604. doi:10.1073/pnas.0702207104. Accessed 30 Aug 2015.

42. Charlesworth B, Barton N. Genome size: does bigger mean worse? Curr Biol: CB. 2004;14(6):233-5. doi:10.1016/j.cub.2004.02.054.

43. Pagel M, Johnstone RA. Variation across species in the size of the nuclear genome supports the junk-DNA explanation for the C-value paradox. Proc Biol Sci/ R Soc. 1992;249(1325):119-24. doi:10.1098/rspb.1992.0093.

44. Wyngaard GA, Rasch EM, Manning NM, Gasser K, Domangue R. The relationship between genome size, development rate, and body size in copepods. Hydrobiologia. 2005;532(1-3):123-37. doi:10.1007/s10750-004-9521-5. Accessed 30 Aug 2015.

45. Hasson E, Naveira H, Fontdevila A. The breeding sites of Argentinian cactophilic species of the Drosophila mulleri complex. Revista Chilena de Historia natural. 1992;65(3):319-26. Accessed 30 Aug 2015.

46. Ruiz A, Cansian AM, Kuhn GC, Alves MA, Sene FM. The Drosophila serido speciation puzzle: putting new pieces together. Genetica. 2000;108(3):217-27.

47. Oliveira DCSG, Almeida FC, O'Grady PM, Armella MA, DeSalle R, et al. Monophyly, divergence times, and evolution of host plant use inferred from a revised phylogeny of the Drosophila repleta species group. Mol Phylogenetics Evol. 2012;64(3):533-44. doi:10.1016/j.ympev.2012.05.012.

48. Fellows D, Heed W. Factors affecting host plant selection in desert-adapted Cactiphilic Drosophila. Ecology. 1972;53(5):850. doi:10.2307/1934300. WOS:A1972N884000008.

49. Heed W, Mangan R. Community ecology of the Sonoran desert Drosophila. In: The Genetics and Biology of Drosophila. London: Academic Press; 1986. p. 311-45.

50. Ruiz A, Heed WB. Host-plant specificity in the Cactophilic Drosophila mulleri species complex. J Anim Ecol. 1988;57(1):237-49. doi:10.2307/4775. Accessed 30 Aug 2015.

51. Etges W, Johnson W, Duncan G, Huckins G, Heed W. Ecological genetics of cactophilic Drosophila. In: Ecology of Sonoran Desert Plants and Plant Communities. Tucson (AZ): University of Arizona Press; 1999. p. 164-214.

52. Comeron JM, Ratnappan R, Bailin S. The many landscapes of recombination in Drosophila melanogaster. PLoS Genet. 2012;8(10): 1002905. doi:10.1371/journal.pgen.1002905. Accessed 31 Aug 2015.

53. Kaminker JS, Bergman CM, Kronmiller B, Carlson J, Svirskas R, et al. The transposable elements of the Drosophila melanogaster euchromatin: a genomics perspective. Genome Biol. 2002;3(12):0084.

54. Rizzon C, Marais G, Gouy M, Biémont C. Recombination rate and the distribution of transposable elements in the Drosophila melanogaster genome. Genome Res. 2002;12(3):400-7. doi:10.1101/gr.210802. Article published online before print in February 2002.

55. Petrov DA, Fiston-Lavier AS, Lipatov M, Lenkov K, González J. Population genomics of transposable elements in Drosophila melanogaster. Mol Biol Evol. 2011;28(5):1633-44. doi:10.1093/molbev/msq337.

56. Kofler R, Betancourt AJ, Schlötterer C. Sequencing of pooled DNA samples (Pool-Seq) uncovers complex dynamics of transposable element insertions in Drosophila melanogaster. PLoS Genet. 2012;8(1):1002487. doi:10.1371/journal.pgen.1002487.

57. Barrón MG, Fiston-Lavier AS, Petrov DA, González J. Population genomics of transposable elements in Drosophila. Ann Rev Genet. 2014;48:561-81. doi:10.1146/annurev-genet-120213-092359.

58. Mackay TFC, Richards S, Stone EA, Barbadilla A, Ayroles JF, et al. The Drosophila melanogaster genetic reference panel. Nature. 2012;482(7384):173-8. doi:10.1038/nature10811. 
59. Casals F, Cáceres M, Manfrin MH, González J, Ruiz A. Molecular characterization and chromosomal distribution of Galileo, Kepler and Newton, three foldback transposable elements of the Drosophila buzzatii species complex. Genetics. 2005;169(4):2047-59. doi:10.1534/genetics. 104.035048 .

60. Casals F, González J, Ruiz A. Abundance and chromosomal distribution of six Drosophila buzzatii transposons: BuT1, BuT2, BuT3, BuT4, BuT5, and BuT6. Chromosoma. 2006;115(5):403-12. doi:10.1007/s00412-006-0071-7.

61. Leung W, Shaffer CD, Reed LK, Smith ST, Barshop W, Dirkes W, et al. Drosophila Muller F elements maintain a distinct set of genomic properties over 40 million years of evolution. G3: Genes|Genomes| Genetics. 2015;5(5):719-40. doi:10.1534/g3.114.015966. Accessed 13 Sept 2015.

62. Schafer DJ, Fredline DK, Knibb WR, Green MM, Barker JSF. Genetics and linkage mapping of Drosophila buzzatii. J Heredity. 1993;84(3):188-94. Accessed 13 Sept 2015.

63. Staten R, Schully SD, Noor MA. A microsatellite linkage map of Drosophila mojavensis. BMC Genet. 2004;5(1):12. doi:10.1186/1471-2156-5-12. Accessed 13 Sept 2015.

64. Bartolomé C, Maside X, Charlesworth B. On the abundance and distribution of transposable elements in the genome of Drosophila melanogaster. Mol Biol Evol. 2002;19(6):926-37.

65. Cridland JM, Macdonald SJ, Long AD, Thornton KR. Abundance and distribution of transposable elements in two Drosophila QTL mapping resources. Mol Biol Evol. 2013;30(10):2311-27. doi:10.1093/molbev/ mst129.

66. Natali L, Cossu RM, Barghini E, Giordani T, Buti M, et al. The repetitive component of the sunflower genome as shown by different procedures for assembling next generation sequencing reads. BMC Genomics. 2013;14:686. doi:10.1186/1471-2164-14-686.

67. Celniker SE, Wheeler DA, Kronmiller B, Carlson JW, Halpern A, et al. Finishing a whole-genome shotgun: release 3 of the Drosophila melanogaster euchromatic genome sequence. Genome Biol. 2002;3(12): 0079.

68. Fiston-Lavier AS, Carrigan M, Petrov DA, González J. T-lex: a program for fast and accurate assessment of transposable element presence using next-generation sequencing data. Nucleic Acids Res. 2011;39(6):36. doi:10.1093/nar/gkq1291.

69. Jiang C, Chen C, Huang Z, Liu R, Verdier J. ITIS, a bioinformatics tool for accurate identification of transposon insertion sites using next-generation sequencing data. BMC Bioinforma. 2015;16:72. doi:10.1186/s12859-015-0507-2.

70. Labrador M, Fontdevila A. High transposition rates of Osvaldo, a new Drosophila buzzatii retrotransposon. Mol Gen Genet: MGG. 1994;245(6): 661-74.

71. García Guerreiro MP, Fontdevila A. Molecular characterization and genomic distribution of Isis: a new retrotransposon of Drosophila buzzati. Mol Genet Genomics: MGG. 2007;277(1):83-95. doi:10.1007/s00438-0060174-0.

72. Xiong Y, Burke WD, Jakubczak JL, Eickbush TH. Ribosomal DNA insertion elements $\mathrm{R} 1 \mathrm{bm}$ and $\mathrm{R} 2 \mathrm{bm}$ can transpose in a sequence specific manner to locations outside the 28s genes. Nucleic Acids Res. 1988;16(22): 10561-73. Accessed 09 Oct 2015.

73. Jakubczak JL, Zenni MK, Woodruff RC, Eickbush TH. Turnover of R1 (type I) and R2 (type II) retrotransposable elements in the ribosomal DNA of Drosophila melanogaster. Genetics. 1992;131(1):129-42. Accessed 19 Oct 2015.

74. Bergman CM, Quesneville H, Anxolabéhère D, Ashburner M. Recurrent insertion and duplication generate networks of transposable element sequences in the Drosophila melanogaster genome. Genome Biol. 2006;7(11):112. doi:10.1186/gb-2006-7-11-r112.

75. Sackton TB, Kulathinal RJ, Bergman CM, Quinlan AR, Dopman EB, Carneiro M, Marth GT, Hartl DL, Clark AG. Population genomic inferences from sparse high-throughput sequencing of two populations of Drosophila melanogaster. Genome Biol Evol. 2009;1:449-65. doi:10.1093/gbe/evp048.

76. Yang HP, Barbash DA. Abundant and species-specific DINE-1 transposable elements in 12 Drosophila genomes. Genome Biol. 2008;9(2):39. doi:10.1186/gb-2008-9-2-r39.
77. Cáceres M, Puig M, Ruiz A. Molecular characterization of two natural hotspots in the Drosophila buzzatii genome induced by transposon insertions. Genome Res. 2001;11(8):1353-64. doi:10.1101/gr.174001.

78. Delprat A, Negre B, Puig M, Ruiz A. The transposon Galileo generates natural chromosomal inversions in Drosophila by ectopic recombination. PloS ONE. 2009;4(11):7883. doi:10.1371/journal.pone.0007883.

\section{Submit your next manuscript to BioMed Central and we will help you at every step:}

- We accept pre-submission inquiries

- Our selector tool helps you to find the most relevant journal

- We provide round the clock customer support

- Convenient online submission

- Thorough peer review

- Inclusion in PubMed and all major indexing services

- Maximum visibility for your research

Submit your manuscript at www.biomedcentral.com/submit

C) BioMed Central 